\title{
Les plans climat énergie territoriaux : voies d'appropriation du facteur 4 par les collectivités et les acteurs locaux?
}

How are sustainable energy action plans means of taking into account the GHG 2050 target by local authorities and local stakeholders

\section{Sylvain Godinot}

\section{OpenEdition \\ Journals}

Édition électronique

URL : http://journals.openedition.org/developpementdurable/8874

DOI : 10.4000/developpementdurable.8874

ISSN : 1772-9971

Éditeur

Association DD\&T

Référence électronique

Sylvain Godinot, «Les plans climat énergie territoriaux : voies d'appropriation du facteur 4 par les collectivités et les acteurs locaux? », Développement durable et territoires [En ligne], Vol. 2, n 1 | Mars 2011, mis en ligne le 31 mars 2011, consulté le 02 mai 2019. URL : http://journals.openedition.org/ developpementdurable/8874; DOI : 10.4000/developpementdurable.8874

Ce document a été généré automatiquement le 2 mai 2019.

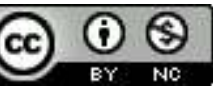

Développement Durable et Territoires est mis à disposition selon les termes de la licence Creative Commons Attribution - Pas d'Utilisation Commerciale 4.0 International. 


\section{Les plans climat énergie territoriaux : voies d'appropriation du facteur 4 par les collectivités et les acteurs locaux?}

How are sustainable energy action plans means of taking into account the GHG 2050 target by local authorities and local stakeholders

Sylvain Godinot

1 Cet article explore la façon dont les collectivités locales françaises ont progressivement pris en compte les enjeux climat énergie et intègrent aujourd'hui l'objectif facteur 4 à travers la démarche des plans climat énergie territoriaux (PCET). Il analyse la rupture que constitue le choix de cet objectif facteur 4 et les difficultés de sa mise en œuvre et explore les pistes actions permettant de les dépasser.

2 Il s'appuie sur des retours d'expérience de l'auteur dans l'accompagnement de plans climat locaux de diverses collectivités, complété de lectures et d'échanges avec des acteurs spécialisés dans la gestion énergétique des collectivités.

3 La première partie retrace l'historique des PCET, du choc pétrolier de 1979 au printemps 2010. La deuxième partie examine les difficultés opérationnelles et stratégiques rencontrées par la première génération de ces plans. La troisième partie propose de mieux articuler plans d'action de court terme, planification territoriale et prospective énergétique, afin que les acteurs locaux s'approprient concrètement l'objectif facteur 4. 


\section{Des premières démarches territoriales sur l'énergie à la première génération de plans climat énergie territoriaux}

\subsection{Des années 1980 aux années 2000 , l'émergence des politiques locales de maîtrise de l'énergie}

$4 \mathrm{Au}$ sortir des trente glorieuses fondées sur l'explosion du recours aux combustibles fossiles, la France est marquée par une très forte centralisation de la production et distribution d'énergie, à travers ses entreprises publiques nationales: EDF, GDF, Elf, Charbonnages de France, AREVA, Commissariat à l'énergie atomique. La rupture des deux chocs pétroliers de 1973 et 1979 ne remet pas en cause cette centralisation, mais initie la mise en place de politiques d'économies d'énergie au niveau national comme dans les collectivités. À partir des années 1980, l'Agence Française de la Maîtrise de l'Énergie, ancêtre de l'Agence de l'environnement et de la maitrise de l'énergie (ADEME), accompagne ces dernières dans une démarche qui deviendra les chartes «Cité Vie » lors de la décennie suivante (Leray, de La Roncière, 2003). Le contre-choc pétrolier de 1986 constitue un coup d'arrêt et entraîne une petite décennie de pause pour les politiques énergétiques (Lenoir, 2007).

Mais la remontée des cours du pétrole à la fin des années 1990 relance l'intérêt des acteurs publics et repositionne l'ADEME sur l'énergie. L'approche énergie des collectivités, à quelques exceptions près, se centre sur la maîtrise de la facture de la collectivité et donc sur le patrimoine municipal et les flottes de véhicules, voire les réseaux de chaleur. Besançon et Montpellier font figure d'exemples (Énergie Cités, 2004) grâce à des réductions de consommation de $30 \%$ à $40 \%$ sur vingt ans dans leur domaine de compétences. Parallèlement, l'intérêt grandissant des collectivités pour l'environnement aboutit à la mise en place de chartes d'écologie urbaine ou de l'environnement, qui seront plus tard intégrées dans le processus des Agendas 21. Ces chartes n'affichent généralement pas d'objectifs quantitatifs globaux d'économies d'énergie, mais des premiers objectifs territoriaux sur les énergies renouvelables apparaissent. Ainsi, le Grand Lyon se dote en 1997 d'un objectif de $15 \%$ d'énergies renouvelables à l'horizon 2010 (Grand Lyon, 1998).

6 Les acteurs locaux du champ des économies d'énergie se professionnalisent au tournant des années 2000 : premières Agences Locales de l'Énergie, développement des associations Amorce et Énergie Cités. Les Espaces Info Énergie (EIE) sont créés avec l'appui de l'ADEME pour sensibiliser le grand public (Métreau, Tillerson, 2007). Dès 1999, des Assises nationales de l'Énergie rassemblent chaque année à Grenoble ou à Dunkerque les collectivités françaises et leurs réseaux de partenaires sur ce sujet.

7 Au plan international, la mobilisation contre le changement climatique s'institutionnalise dans les années 1990 par la conférence de Rio, puis par le protocole de Kyoto, par lequel les États industrialisés s'engagent à traduire leurs objectifs en plans d'actions nationaux. Si la prospective française intègre l'énergie presque dès ses débuts (Berger, 1967; Commissariat Général du Plan, 1972) elle reste d'échelle nationale. Les travaux du Commissariat général au Plan proposent en 1998 trois scénarios pour 2010 et 2020 dont celui nommé S3, «État protecteur de l'environnement » qui vise à atteindre l'objectif de 
Kyoto (Commissariat Général du Plan, 1998; Global Chance, 2003). Au départ, l'État français fait à peine appel aux collectivités territoriales pour contribuer à la succession de plans climat nationaux, plutôt sectoriels (PNLES des années 1990, PNLCC de 2000). L'outil plan climat énergie territorial apparaît en germe dans le « Mémento des décideurs publié en 1999 par la Mission Interministérielle à l'Effet de Serre (MIES), mais naît formellement avec le Plan Climat de 2004 et s'affirme avec son actualisation de 2006 puis 2010 (MEEDD, 2004 ; MEEDD, 2006 ; MEEDDM, 2010 ; Berthier, Richard, 2010).

La préoccupation climatique s'affirme progressivement dans le cadre réglementaire national avec la loi sur l'Air et l'Utilisation Rationnelle de l'Énergie de 1996, qui définit les gaz à effet de serre (GES) comme des polluants et rend les plans de déplacement urbains obligatoires pour les agglomérations de plus de 100000 habitants, mais ne fixe pas d'objectifs climatiques. Par contre, une circulaire ministérielle d'août 1999 invite les préfets à inclure les objectifs de Kyoto dans la quatrième génération de contrats de plan État Régions (CPER 2000-2006). La loi solidarité et renouvellement urbains (SRU) de 2000 permet également de mieux intégrer les enjeux environnementaux dans les documents d'urbanisme. L'enjeu du risque climatique vient dès lors compléter, voire supplanter, celui de la vulnérabilité financière découlant de la dépendance énergétique de la France.

C'est avec la loi d'aménagement et de développement durable du territoire de 1999, dite "loi Voynet", qu'apparaissent les schémas de service collectif de l'énergie, outils de planification régionale à vingt ans. Ils seront abandonnés suite à l'alternance gouvernementale de 2002. Avec l'appui des services de l'État, certaines régions mettent en conséquence en place en 2002 des observatoires régionaux de l'énergie et des gaz à effet de serre (RARE, 2004). Ces schémas de service collectif sont peut-être les premiers exercices de prospective énergétique territoriale française.

\subsection{0-2008, les premiers plans climat locaux : intégration de l'objectif de Kyoto et développement d'outils méthodologiques}

10 Dès 2002, l'agglomération grenobloise et la ville de Chalon-sur-Saône font partie des collectivités pionnières qui s'affichent sur le changement climatique et participent au groupe de travail national ad hoc du Plan climat. Elles élaborent les premiers plans climat qui se fondent sur les objectifs de Kyoto. Ces plans intègrent des actions internes ainsi qu'une sensibilisation des acteurs par une approche partenariale.

11 En février 2003, le gouvernement français déclare vouloir s'inscrire dans la trajectoire du «facteur 4 », objectif qui sera inscrit dans le plan climat national de 2004, puis dans la loi d'orientation et de planification sur l'énergie de 2005.

12 L'ADEME teste dès 2005 puis diffuse en 2007 l'outil «Bilan Carbone Collectivités » qui devient la référence pour établir le diagnostic initial des émissions de GES du territoire et de la collectivité en tant qu'institution. Les émissions du territoire y sont réparties en trois périmètres concentriques - institution, politiques publiques, territoire. Cette approche légitime le rôle de la collectivité comme pilote d'une démarche collective et volontariste (ADEME, 2006). Les premiers bilans carbone confirment que la collectivité ne contrôle en maîtrise directe que quelques pourcents des émissions de gaz à effet de serre qui ont lieu sur son territoire (patrimoine bâti, véhicules, achats, etc.) et quelques dizaines de pourcents en incluant ses politiques publiques (habitat, transports en commun...). Si elle doit s'efforcer d'optimiser ses émissions directes, elle doit surtout 
développer des politiques publiques qui aident les habitants à être sobres en carbone, notamment par le biais de la planification territoriale. Elle doit de plus piloter une dynamique partenariale afin d'inciter les autres acteurs du territoire (autres collectivités, entreprises, citoyens...) à contribuer à l'effort et à évoluer vers des modes de production et de consommation plus sobres et plus durables. Cette réalité l'invite à sortir de son rôle de donneur d'ordres pour aller vers celui d'animateur territorial.

Pour aller au-delà du bilan carbone et décliner le plan climat national, l'ADEME publie en 2005 le guide : Un plan climat à l'échelle de mon territoire. Celui-ci intègre les préoccupations énergétiques classiques mais aussi d'autres domaines plus spécifiques au climat comme celui de la biodiversité (adaptation) et de l'agriculture (GES hors CO2). Le plan climat territorial, qui ne dispose pas encore d'une définition légale ou réglementaire, y est caractérisé par des objectifs quantitatifs et datés de réduction de GES sur un territoire donné, le pilotage d'un ensemble d'acteurs par une collectivité, et un plan d'action incluant éventuellement un volet adaptation (ADEME, 2005).

À la même période, les exercices de planification territoriale réglementaire (schémas de cohérence territoriale ou SCOT, plans locaux d'urbanisme - PLU - plans de déplacements urbains - PDU...) se renforcent et commencent pour certains à intégrer un objectif climatique allant parfois pour certains jusqu'à celui du facteur 4. L'intégration de ces objectifs semble cependant avoir des effets assez limités : une évaluation par l'association ETD conclut que "les enjeux énergétiques et climatiques n'impactent pas les stratégies des ScoT " (ETD, 2009). Selon ce document, "l'analyse traduit l'absence relative de réflexion prospective sur les perspectives d'évolution du territoire, en fonction des grandes tendances identifiées, des contraintes à venir et des potentialités locales. Les perspectives de la raréfaction et du coût des énergies fossiles, ainsi que du changement climatique ne sont pas envisagées comme des facteurs d'évolution majeurs susceptibles de modifier en profondeur l'organisation du territoire. "

Différents exercices de prospective nationale énergie-climat ont lieu pendant cette période. Ceux de la fin de la décennie 1990 ont servi de référence dans des exercices de prospective énergie régionaux même s'ils sont restés assez méconnus (Global Chance, 2003). C'est aussi l'époque de la naissance du concept de facteur 4 (Weizsäcker et al., 1997). La mission «facteur 4 » de 2006, dans le cadre de la future loi sur l'énergie, permet de synthétiser les quelques rapports français qui intègrent l'enjeu climatique au niveau du facteur 4 et plus seulement de l'objectif de Kyoto (de Boissieu, 2006). Six modèles et scénarios y sont identifiés, dont celui du Ministère de l'Industrie (Enerdata-LEPPI, 2005), celui de l'association négaWatt (Association Négawatt, 2005) et celui de la Mission Interministérielle à l'Effet de Serre - MIES - (Radanne, 2005). Ce dernier mentionne les collectivités sans leur accorder beaucoup de place et recommande de les inciter à bien intégrer l'enjeu climatique dans l'urbanisme. Mais ces exercices de prospective énergétique nationale sont peu cités dans les plans climat territoriaux. Prospective énergie-climat nationale, planification territoriale et plans climat territoriaux se juxtaposent encore de façon quasiment étanche.

\subsection{Depuis 2007, le Grenelle de l'Environnement : généralisation des plans climat énergie territoriaux, et objectifs $3 \times 20 \%$ en 2020}

L'appellation «plans climat énergie territoriaux» (PCET) est consacrée en 2007 par le Grenelle de l'environnement. La loi Grenelle 1 (Grenelle 1, 2009) incite les collectivités à 
en réaliser, la loi Grenelle 2 (Grenelle 2, 2010) les rend obligatoires pour les collectivités de plus de 50.000 habitants mais en donne une définition encore large :

"Art. L. 229-26. - I. - Les régions et la collectivité territoriale de Corse, si elles ne l'ont pas intégré dans le schéma régional du climat, de l'air et de l'énergie mentionné à l'article L. 222-1, les départements, les communautés urbaines, les communautés d'agglomération ainsi que les communes et les communautés de communes de plus de 50000 habitants doivent avoir adopté un plan climat-énergie territorial pour le 31 décembre 2012

"Lorsque ces collectivités publiques s'engagent dans l'élaboration d'un projet territorial de développement durable ou Agenda 21 local, le plan climat-énergie territorial en constitue le volet climat.

«II. - En tenant compte des bilans des émissions de gaz à effet de serre prévus à l'article L. 229-25, ce plan définit, dans les champs de compétences respectifs de chacune des collectivités publiques énumérées au I du présent article:

" $1^{\circ}$ Les objectifs stratégiques et opérationnels de la collectivité afin d'atténuer et lutter efficacement contre le réchauffement climatique et de s'y adapter;

" $2^{\circ}$ Le programme des actions à réaliser afin notamment d'améliorer l'efficacité énergétique, d'augmenter la production d'énergie renouvelable et de réduire l'impact des activités en termes d'émissions de gaz à effet de serre, conformément aux objectifs issus de la législation européenne relative à l'énergie et au climat ;

« $3^{\circ}$ Un dispositif de suivi et d'évaluation des résultats. » (Article 75).

L'article 69 de cette loi définit également les schémas régionaux climat air énergie (SRCAE) qui constituent des exercices obligatoires de planification et de prospective énergie-climat territoriale, plus larges que les anciens schémas de service collectifs de l'énergie des lois Voynet et Pasqua. Bien qu'associant les Régions, les SRCAE placent l'État en position d'acteur clé dans la planification énergétique territoriale. Par ailleurs, la refonte du Ministère en charge de l'environnement et du développement durable et sa fusion avec celui de l'équipement, modifie en profondeur le rôle des services déconcentrés de l'État. Elle aboutit à la création de nouvelles missions «Grenelle » dans les directions régionales de l'environnement, de l'aménagement et du logement (DREAL, ex DRIRE) et les directions départementales du territoire (DDT, ex DDE), qui se mettent progressivement en place à partir de début 2009 et se préparent à construire les SRCAE et à contrôler les plans climat obligatoires (MEEDDAT, 2009).

19 La dynamique du Grenelle, malgré la lenteur dans l'adoption du projet de loi Grenelle 2 et de ses décrets d'application, met en mouvement une grande partie des collectivités. L'observatoire des PCET piloté par l'ADEME recense, à son ouverture en mars 2010, 198 PCET en élaboration ou en vigueur. Moins du quart d'entre eux sont opérationnels, la collectivité ayant voté un plan d'action sur son patrimoine et ses services publics et ayant engagé une démarche partenariale.

Début 2009, L'ADEME propose une méthodologie détaillée des PCET dans son Guide méthodologique: construire et mettre en cuvre un plan climat territorial (ADEME, 2009). La démarche proposée s'inspire de celle des Agendas 21 dans ses étapes et sa méthodologie, et nombre de collectivités intègrent d'ailleurs le plan climat comme un des cinq axes de leur agenda 21, conformément au cadre de référence du ministère. C'est par exemple le cas du Grand Lyon dans son Agenda 21 2007-2009. Par ailleurs, l'ADEME introduit en France la labellisation Cit'ergie, outil européen qui évalue de façon indépendante la démarche de politique énergétique ou climatique d'une ville ou d'intercommunalité, sur la base de 87 actions prédéfinies qui visent à évaluer sous l'angle énergétique la plupart 
de ses champs d'actions. Fin 2009, quatre PCET sont certifiés Cit'ergie, et une douzaine d'autres collectivités sont en cours de certification. Par comparaison, environ 600 Agendas 21 de collectivités sont identifiés début 2010, dont 141 labellisés par le Ministère de l'environnement et du développement durable mi 2010 .

Bien que le Grenelle n'impose pas d'objectifs quantitatifs aux collectivités, la plupart de celles qui ont choisi de s'en fixer ont repris à leur compte les objectifs à 2012 assignés à la France par le protocole de Kyoto, ou à moyen terme par l'Europe pour 2020 (les «3x20\%»). Début 2010, 104 collectivités françaises ont ainsi signé la Convention des Maires qui promeut cet objectif. Pour le long terme, ces collectivités reprennent le plus souvent l'objectif facteur 4 en 2050.

Dans les suites du Grenelle, la cellule prospective du ministère du développement durable popularise le concept de prospective énergétique et de ville post-carbone, en lançant dans appels à projets sur le sujet. Cette première génération de démarches territoriales climaténergie va être confrontée à des difficultés allant au-delà de celles déjà expérimentées par Agendas 21. Il leur faut en effet adopter un objectif quantifié et daté, ambitieux, s'appliquant à tout le territoire et imposant des mutations structurelles de moyen et long terme. Les collectivités, plus habituées à créer dans le court terme des institutions basées sur les grands principes (Lorrain 2004), ont en effet du mal à s'attaquer à la réduction des GES qui exige à la fois un engagement de long terme et une des réductions quantifiées à court terme.

\section{Dépasser les écueils rencontrés par la première génération de plans climat énergie territoriaux}

L'élaboration des plans climat conduit les collectivités à devoir gérer des difficultés de plusieurs ordres : planifier des actions dans un cadre légal et un jeu d'acteurs publics mouvants; évaluer les émissions du territoire malgré d'importantes difficultés méthodologiques; formaliser et prioriser un plan d'action énergie climat et l'intégrer dans les arbitrages sur les moyens; faire évoluer leurs compétences et champs d'intervention comme acteur public auprès des partenaires du territoire. Il semble exister un gouffre entre l'investissement politique nécessaire pour résoudre la crise climatique et les marges de manœuvre à la disposition des élus.

\subsection{S'évaluer : choisir le périmètre et les secteurs à quantifier}

Le choix d'un objectif territorial quantitatif et daté constitue une rupture par rapport à la logique d'amélioration, continue mais relative, des Agendas 21 territoriaux qui sont également en plein essor. Dans un plan climat, le diagnostic de départ sert notamment à identifier le niveau d'émissions initial, et à déduire les points d'étape visés à des horizons de temps allant de quelques années à plusieurs décennies.

L'une des forces de l'approche climatique réside donc dans la lisibilité de l'indicateur « unique » que constitue la tonne de $\mathrm{CO}_{2}$ (gaz carbonique) évitée, qui permet de comparer une politique de transports à un investissement de chaufferie au bois, malgré le caractère forcément très réducteur de cette analyse. Cette simplicité de l'indicateur des tonnes de GES ne doit pas amener à comparer abusivement les territoires (émissions par habitant) sans expliciter les méthodologies et périmètres de comptage, ainsi que les différences de 
compétences qui déterminent en première approche les émissions directes de deux collectivités. La comptabilisation des émissions ne peut se restreindre à celles qui sont liées aux compétences de la collectivité; ceci l'inviterait à limiter ses champs d'intervention, alors que leur développement pourrait réduire les émissions du territoire desservi. Ainsi, développer les lignes de bus augmente les émissions de la collectivité, mais réduit globalement celles du territoire en permettant aux habitants de ne pas recourir à des voitures plus émettrices. D'autre part, d'autres enjeux sont liés au PCET comme la santé (qualité de l'air, lutte contre la précarité énergétique) ou d'autonomie énergétique et ils ne peuvent être intégrés dans l'indicateur de $\mathrm{CO}_{2}$ évité.

Deux méthodologies existent pour comptabiliser les émissions de GES d'un territoire. La première est l'approche par le bilan carbone de l'ADEME. On prend en compte toutes les émissions liées aux différents postes d'un produit ou service dans une logique d'analyse $\mathrm{du}$ cycle de vie, et on les impute à la fois au producteur et au consommateur. Ainsi, une ville qui construit un bâtiment intègre dans son bilan carbone interne les émissions liées à la fabrication du ciment, et les comptera à nouveau dans son bilan territorial si la cimenterie est sur son territoire. La seconde approche, par un inventaire cadastral - qui correspond pour l'État aux engagements de Kyoto par exemple - évite ce double comptage en affectant la responsabilité des émissions à l'émetteur, ce qui conduit à négliger toutes les importations d'énergie et de matériaux car elles n'ont pas lieu sur son territoire. Les villes étant par essence importatrices de nourriture et d'énergie, un inventaire de GES sera d'autant plus sous-estimé que la ville sera résidentielle et tertiaire. Les territoires ruraux ou industriels se voient affecter l'essentiel de ces émissions. Une solution pour éviter la tentation de la délocalisation serait de faire évoluer les inventaires pour qu'ils intègrent des importations et exportations et en partagent la responsabilité avec les territoires concernés.

Pour s'évaluer plus qualitativement, les collectivités disposent depuis 2008 de l'outil européen Cit'ergie, et depuis 2009 du portail internet des PCET de l'ADEME. Le décret PCET découlant du Grenelle 2, à paraître fin 2010, pourrait apporter un cadre plus homogène. Les associations environnementales n'ont à ce jour pas noté les PCET qu'elles jugent sérieux ni dénoncé ceux de complaisance.

\subsection{Clarifier les rôles en évolution des différents acteurs publics et leur articulation}

Les orientations de la loi Grenelle 2 ont un impact important sur les politiques d'urbanisme, d'habitat et de transports sous l'angle climat énergie (Boutaud, 2009). La loi Grenelle 2 impose aux collectivités jugées prioritaires de publier leur plan climat avant fin 2012, puis de le réviser au moins tous les cinq ans. Celles-ci doivent concilier cette échéance avec le rythme pluriannuel des différents exercices de planification urbaine (PLU, SCOT, etc.). L'intégration des contraintes climat énergie dans leurs politiques d'aménagement et d'urbanisme prendra donc encore des années, selon le rythme de révision de ces documents.

29 Faire face aux défis énergétique et climatique requiert une intervention forte des pouvoirs publics, ce qui constitue une nouvelle mission de service public encore peu définie. Le cadre de leur intervention apparaît d'autant plus en évolution que la réforme générale des politiques publiques devrait profondément remodeler les compétences des collectivités (création de l'échelon des métropoles, remise en cause de la clause de 
compétence générale des régions et départements) et qu'elles s'inquiètent pour leur budget (suppression de la taxe professionnelle, dépendance accrue envers les financements de l'État).

Par ailleurs, le télescopage des rôles entre l'État et les collectivités, de même qu'entre les collectivités elles-mêmes, crée des problèmes de lisibilité. Il est alors difficile de mobiliser les acteurs qui interviennent à plusieurs échelles territoriales, sur des compétences réparties entre plusieurs collectivités. Ainsi, pour les logements anciens dégradés, si la lutte contre la précarité énergétique relève principalement des départements, la politique de rénovation de l'habitat privé revient souvent aux intercommunalités et aux communes (PUCA, 2009). De même, dans le domaine de l'habitat social, les régions interviennent souvent en complément des intercommunalités.

\subsection{Adapter les objectifs climat énergie aux potentiels des territoires et à l'évolution des connaissances climatiques}

31 L'objectif de réduction des GES (à hauteur des 3x20 et du facteur 4) semble aujourd'hui devoir s'imposer à tous les territoires. En effet, le territoire qui viserait un objectif inférieur afficherait publiquement son choix de faire «moins que sa part », ce qui est politiquement difficile. Celui qui afficherait son intention de faire mieux ou plus vite devrait produire un plan d'action très solide pour éviter de sembler irréaliste ou velléitaire. En pratique, il est cependant indispensable que l'objectif national soit réparti selon les responsabilités et capacités des territoires, comme l'a été l'objectif européen pour Kyoto entre les pays membres de l'Union Européenne. L'atteinte par la France de son objectif d'énergies renouvelables ne sera ainsi possible que si des régions rurales acceptent de dépasser largement le seuil de 23 \%, grâce à l'éolien par exemple, en accord avec les aires urbaines à moindre potentiel.

32 L'horizon de temps des plans climat s'est par ailleurs éloigné : de l'ordre de la décennie pour atteindre l'objectif de Kyoto à celui d'une quarantaine d'années pour le facteur 4 . Il convient néanmoins de rappeler que cet objectif de facteur 4 n'est pas absolu. C'est l'une des traductions du seuil critique de réchauffement à $+2^{\circ} \mathrm{C}$ proposé par le groupe d'experts intergouvernemental sur l'évolution du climat - GIEC (IPCC, 2007), qui situe la fourchette entre le facteur 4 et le facteur 14, soit une division mondiale par 2 à 7 en 2050. Atteindre le facteur 4 ne donne qu'une chance sur deux de limiter le réchauffement à $+2^{\circ} \mathrm{C}$, ce qui est l'objectif que s'est fixé l'Union européenne (de Boissieu, 2006).

La nécessité d'un horizon à moyen terme crée également un débat sur le point d'étape en 2020 : $-20,-30$, ou $-40 \%$ ? L'Union Européenne s'est engagée unilatéralement à -20\% mais envisage $-30 \%$ en cas d'accord international satisfaisant. Pour les pays développés, une réduction de $-40 \%$ correspondrait mieux aux recommandations du GIEC vu le budget carbone limité dont dispose l'humanité (Meinshausen et al., 2009). S'aligner sur la position prudente du GIEC, soit le facteur 14, ou sur l'objectif de $-40 \%$ en 2020, conduirait à viser le "zéro émission" ou la ville post-carbone, sans recours aux énergies fossiles. Certains chercheurs vont jusqu'à proposer des droits d'émissions négatives dès 2025 (Baer et al., 2008). De tels objectifs ont l'avantage d'exprimer clairement l'ampleur des mutations nécessaires. Si l'année de référence reste 1990 pour l'Europe, elle équivaut à peu près en niveau d'émissions à 2009 pour la France et ne laisse donc que 10 ans pour atteindre l'objectif. 


\subsection{Utiliser tous les leviers disponibles pour mettre en œuvre ces plans d'action : planification territoriale, nouveaux partenariats public privé pour renforcer les moyens humains et financiers et exemplarité} principale de réduire la vulnérabilité économique de la collectivité ou de son territoire aux fluctuations des prix de l'énergie, ou d'anticiper de potentielles obligations réglementaires (cas de la conversion au bois des gros réseaux de chaleur). Mais pour la majorité des actions, l'enjeu climat-énergie s'ajoute comme un objectif secondaire à des missions existantes. Les services de la collectivité doivent par exemple examiner comment produire autant de logements sociaux neufs, de qualité énergétique supérieure (niveau bâtiment basse consommation - BBC - généralisé par la réglementation thermique de 2012) sans financement additionnel. L'ampleur des engagements financiers que demandent les objectifs du plan climat peut faire naître un scepticisme chez des agents conscients de la difficulté à faire évoluer les arbitrages budgétaires. l'énergie pour les années à venir, qui correspond à une approche rationnelle sur le court 
terme, mais qui légitime de ne pas rechercher de transformations structurelles. Par ailleurs, les collectivités disposent de peu d'outils financiers efficaces dans le secteur de l'énergie : faible cours des certificats d'économie d'énergie et des quotas de $\mathrm{CO}_{2}$, projets domestiques anecdotiques, annulation de la taxe carbone. Ils viennent au mieux en addition aux subventions, mais ne permettent qu'exceptionnellement de modifier la rentabilité des choix d'investissements. La collectivité peut néanmoins développer des aides massives sur des politiques innovantes qui sont en dehors de ses compétences traditionnelles. L'agglomération grenobloise consacre à son dispositif de rénovation énergétique des copropriétés - la campagne « Mur Mur »-plusieurs millions d'euros par an. Celui-ci est adossé à un impôt supplémentaire sur les ménages. Qu'une collectivité crée ainsi de nouvelles recettes pour financer de nouvelles dépenses est encore exceptionnel, même si le développement de la tarification du stationnement des voitures a cependant vocation à se généraliser.

41 Pour agir de façon lourde sur les investissements, de nouvelles offres de services se créent, comme la société coopérative d'intérêt collectif (SCIC) d'approvisionnement en bois énergie créée par le Conseil régional Picardie avec d'autres acteurs publics (Départements) et privés (professionnels du bois); les projets de société d'économie mixte sur les énergies renouvelables (Conseil régional Rhône-Alpes, Ville de Paris); les vélos en libre service (Velo'V); les prêts bonifiés (Régions). Il s'agit là de partenariats publics privés qui lui permettent d'élargir ses champs d'intervention sans réviser ses champs de compétences. La collectivité peut aussi accompagner les acteurs de son territoire pour accélérer le changement, en cofinançant l'aide à la décision: conseils d'Espace Info Énergie pour les habitants, offre de services comme les plans de déplacement interentreprises ou les diagnostics énergie groupés en entreprises. Elle mobilise pour cela principalement des ressources humaines, notamment associatives mais également privées. Au stade actuel, elle semble choisir par défaut de ne pas ou peu internaliser ces services, pour des raisons qui seraient à étudier : difficulté due à l'absence de compétence administrative, besoin de faire appel à une expertise encore émergente et plus présente dans le secteur privé, manque de nouvelles ressources pérennes...

\subsection{Animer le jeu d'acteurs locaux, un nouveau rôle pour des élus chefs d'orchestre}

42 Pour le pilote du plan climat, afficher un objectif territorial revient essentiellement à parier sur sa capacité à amener les partenaires locaux à s'engager sur ce même objectif. Certaines collectivités choisissent de distinguer l'objectif interne de celui du territoire (Ville de Paris, Métro de Grenoble), pour souligner cette différence de responsabilité et éventuellement différencier le niveau d'effort. Il revient à l'élu pilote ou au chef de l'exécutif de la collectivité d'engager cette démarche de mobilisation du territoire par des acteurs clé.

43 Cette approche s'amorce par la phase de concertation initiale mais il faut aller au delà. Une bonne sensibilisation sur les enjeux climatiques suffit généralement à faire partager l'intention d'agir à un premier cercle d'acteurs du territoire (cf. cycle de conférences climat du PCET de Paris en 2007). Il faut ensuite formaliser cette intention en les incitant à produire leur propre plan d'action. Selon Pierre Radanne, la démarche collective est indispensable pour que les acteurs prennent conscience qu'ils doivent s'impliquer grâce à 
une saine émulation. Je fais, si tu fais, si nous faisons tous : c'est en tant qu'élément de l'action collective que prend sens l'action individuelle (Radanne, 2005).

Le cadre de partenariat se traduit souvent par des chartes volontaires, comme celles de Mulhouse Alsace Agglomération et de la Métro de Grenoble. Cette dernière innove en demandant à ses partenaires d'évaluer leurs progrès et de tenir des objectifs qu'elle leur fixe. La collectivité contribue ici à faire évoluer les imaginaires en impulsant un débat sur les visions souhaitables $d u$ futur à l'échelle du territoire, donc en facilitant l'appropriation d'enjeux globaux par leur incarnation à une échelle plus concrète. À cette fin, les collectivités peuvent impliquer leurs partenaires en soutenant des projets : travail d'étudiants "Rennes $+6^{\circ} \mathrm{C}$ », appel à projets climat multipartenaires de la Métro de Grenoble, appel à projets de la Région Rhône-Alpes sur l'éco-citoyenneté.

\subsection{La vision développement durable des élus : absente des PCET ?}

Les élus pilotes sont amenés à faire partager une vision d'autant plus concrète et appliquée du développement durable qu'elle est restreinte aux enjeux climat-énergie. Elle les conduit à naviguer entre deux approches: celle de durabilité faible et celle de durabilité forte, selon la distinction faite par Aurélien Boutaud (Boutaud, 2009). Selon les tenants de la première, les objectifs environnementaux seront atteints grâce à la croissance, les solutions sont principalement technologiques, et la stratégie à adopter consiste à faire les ajustements nécessaires. Pour les tenants de la seconde, les objectifs ne seront atteints qu'en incluant un important volet de sobriété voire de décroissance et les solutions sont surtout organisationnelles et politiques, ce qui impose une stratégie de rupture. Ce schisme constitutif du développement durable apparaît dès la naissance du concept avec le cri d'alarme du Club de Rome (Meadows et al., 1972). Ces acteurs se distinguent aussi par leur rapport au principe de précaution, qui s'incarne dans le débat climatique par l'importance accordée au non-franchissement du seuil des $+2^{\circ} \mathrm{C}$, défini comme seuil critique par le GIEC. Pour maximiser le nombre et le poids des acteurs mobilisés, les élus ont intérêt à essayer d'impliquer les tenants des deux approches dans l'action. Boutaud souligne que les décideurs politiques (et économiques) ont pour la plupart en commun un socle idéologique productiviste, qui les conduit à pencher pour la durabilité faible. Toutefois, les écologistes, souvent tenants de l'autre approche, sont surreprésentés parmi les élus en charge du développement durable. Ils doivent alors faire le pont entre leurs alliés politiques et leurs électeurs. Boutaud considère que les plans climat sont l'archétype d'une approche " écolo-techno » de certains agendas 21, qui tend à réduire le développement durable à sa dimension environnementale et réduit la démocratie participative pour une approche plus axée sur la technique et la planification.

La prochaine période, "post-Grenelle », pourrait voir apparaître des plans climat de seconde génération, qui parviendraient à résoudre une partie des difficultés de ceux de la première et sauraient articuler de façon plus cohérente les objectifs affichés à moyen et long terme, et un plan d'action de court terme crédible pour les atteindre. 


\section{Vers des plans climat air énergie de $2^{\mathrm{e}}$ génération : combiner plans d'action, planification et prospective territoriales}

Pour réussir de nouveaux plans climat, plusieurs actions semblent indispensables : agir tout de suite, par des plans d'action transversaux, pour mettre en mouvement les acteurs; articuler la planification territoriale avec celles, sectorielles de l'environnement (eau, déchets, bruit, air...); initier une démarche de prospective territoriale qui permettra d'assimiler l'ampleur du changement requis par le facteur 4 . Ces trois approches devront être menées simultanément et de façon itérative. Pour cela, les plans climat doivent traiter simultanément trois échelles de temps : celle du mandat pour le plan d'action en cours, celle de la décennie ou plus pour la planification et celle du long terme pour la prospective.

\subsection{Généraliser les plans d'action de première génération pour multiplier les dynamiques territoriales}

Les collectivités qui ont engagé une démarche de plan climat ont besoin de temps pour s'imprégner des concepts et des exigences liés à la crise climatique et énergétique. Or les objectifs ambitieux fixés pour l'horizon 2020 doivent être réalisés pendant la durée de deux à trois mandats électifs locaux, y compris celui en cours. Pour les équipes en place, il est donc essentiel de mobiliser les services et d'apprendre en faisant. Cela passe par des actions incontournables comme les audits énergétiques globaux de patrimoine, la programmation des travaux prioritaires, l'introduction de critères carbone dans les marchés publics, un développement massif des modes de transports doux... Pendant cette phase, il est tout aussi important d'initier concrètement la démarche partenariale, car la création de relation de coopération efficaces prend du temps. Enfin, les collectivités les plus importantes ou innovantes devraient se donner un droit d'expérimentation économique, juridique et technique, pour élargir le champ des possibles.

La période actuelle doit aussi favoriser une mobilisation large des citoyens et des acteurs du territoire. Conformément à la méthodologie des agenda 21 , la concertation initiale la plus large possible doit permettre la construction d'un diagnostic partagé, afin de favoriser l'appropriation du sujet et des solutions et de susciter la mobilisation. L'écueil de cette approche peut être de consacrer beaucoup de temps et de ressources humaines pour ne mobiliser au final que les acteurs déjà intéressés (retraités, associations environnementales) et n'identifier que des actions déjà repérées en interne.

Dans ce calendrier contraint, une autre voie consiste à co-construire un état des lieux sommaire et un plan d'actions "de mise en mouvement " en moins d'un an. C'est la démarche qu'initie l'Agence Locale de l'Énergie de Lyon avec les communes périphériques, en lien avec le plan climat de la communauté urbaine. Cette approche vise à faire en sorte que chaque commune s'approprie le sujet par des actions consensuelles, plutôt que par un long diagnostic. Fondée sur une logique tendancielle, elle pourrait se réduire à une action superficielle, d'éco-blanchiment ou "Greenwashing", si la collectivité s'y limite. En menant de front l'action et la réflexion, en complétant le diagnostic à mesure des progrès réalisés, elle cherche à s'inspirer de la théorie de 
l'engagement : les actes entraîneront plus vite des changements de valeur qu'un débat sur la nécessité d'agir, ou qu'un diagnostic même précis. D'autre part, pour la plupart des élus qui ont une vision de "durabilité faible», l'action immédiate "de bon sens", « pragmatique » est facile à décider, alors que le débat sur le facteur 4 - dont nous avons vu précédemment l'échelle quasi-inatteignable - les irrite en remettant trop le système en cause. Enfin, la sociologie de l'environnement montre que l'engagement écologique est lié notamment à la confiance dans les institutions et la démocratie (Bozonnet, 2009). Cette confiance étant particulièrement faible en France par rapport aux autres pays d'Europe de l'Ouest (Algan, Cahuc, 2008), elle doit faire l'objet d'un travail spécifique, notamment par l'exemplarité des acteurs publics.

\subsection{Articuler planification territoriale et planification environnementale sur le volet air énergie climat}

51 La mise en œuvre de ces premiers plans d'actions, composés principalement de mesures correctives périphériques, ne suffira pas pour mettre les collectivités dans la trajectoire des objectifs fixés pour le long terme. Il faudra utiliser les outils des politiques locales d'habitat, d'urbanisme et de transports pour qu'ils contribuent à l'atteinte des objectifs climat énergie. Les lois de décentralisation de 1982 et de 1983 avaient posé les bases de la planification territoriale (plans d'occupation des sols - POS - PDU, programmes locaux de l'habitat - PLH - CPER, schémas directeurs), tandis que les collectivités s'étaient essayé à la prospective territoriale, avec l'appui de la DATAR et du Commissariat au Plan (Durance, 2007 : 16). Mais les deux approches étaient restées disjointes. La nouvelle logique de l'État (SRCAE) cherche donc à imposer l'articulation de la planification territoriale et de ses outils (SCoT, PLU, schéma régionaux d'aménagement et de développement durable du territoire - SRADDT...) avec celle, sectorielle et encore très récente, sur les volets environnementaux, notamment ceux liés aux enjeux air climat énergie. L'intégration systématique de l'énergie dans ces schémas territoriaux apparaît « comme une exigence incontournable» (MEEDDM CGDD, 2009). La révision des documents d'urbanisme constitue l'un des enjeux d'action immédiate (Clerc D et al., 2008), mais il est trop tôt pour dire si le SRCAE saura imposer ses objectifs aux documents d'urbanisme (Cordeau, Cassin, 2009). D'autre part, un décloisonnement entre outils environnementaux se dessine, par l'absorption des plans régionaux de qualité de l'air (PRQA) par les SRCAE pour gérer un compromis entre des enjeux parfois contradictoires, comme la qualité de l'air locale (pollution aux particules notamment) et globale (GES). Les outils d'évaluation tels que les observatoires régionaux prendront ici tout leur sens. L'une des difficultés qu'introduit cette intégration de l'environnement est de passer en vision systémique (approche qui traite de tous les enjeux du territoire) et non plus discontinue, par thématique. Outre l'urbanisme, l'habitat et les transports, sur lesquels les collectivités tiennent un rôle de premier plan, la production et la distribution d'énergie constituent un enjeu à reconquérir pour les collectivités. La planification énergétique territoriale (production et distribution d'énergie mais aussi gestion de la demande) constituera un élément clé des plans climat de deuxième génération. En effet, articuler les réseaux de distribution d'électricité, de gaz et de chaleur contribuera à la réussite de ces plans. L'effacement des pointes de consommation électrique (réseaux intelligents ou "smart grids") ou la pénétration de la cogénération limiteront le recours aux centrales thermiques. Cette articulation entre planification territoriale et énergétique conduira à repenser l'échelonnement des actions. On évitera de convertir un réseau de chaleur du charbon au 
gaz plutôt que d'attendre quelques années de plus pour l'alimenter directement au bois, car on risquerait d'atteindre l'objectif de $-20 \%$ en 2020 mais de devoir réinvestir pour le facteur 4.

C'est à cette condition que les collectivités pourront avoir un impact fort sur le deuxième cercle du bilan carbone - au-delà de leur patrimoine propre - celui des politiques publiques comme l'habitat, les transports ou l'aménagement du territoire. L'intégration du climat dans ces politiques publiques permettra à la collectivité de changer d'échelle et de renforcer la part du budget " orientée climat » : de quelques pour mille dans les plans d'action de première génération à plusieurs pourcents, ce qui est l'ordre de grandeur préconisé par le rapport Stern (Stern, 2006). Dans ce cadre, des réaffectations importantes des budgets d'investissement pourraient être déclinées conformément aux appels à « un effort de guerre » ou un « plan Marshall du climat ». Ce niveau d'ambition semble être le seul à même d'inverser la tendance mondiale des émissions avant 2015, c'est-à-dire durant le mandat actuel des élus municipaux français.

53 L'exercice de prospective doit permettre de convaincre collectivités et partenaires de la nécessité de ce changement d'échelle.

\subsection{Développer une prospective territoriale énergie climat}

À notre connaissance, aucun plan climat énergie territorial n'a à ce jour conçu de scénarios multiples en fonction de différents modèles d'évolution des prix des énergies ou du carbone. On peut donc considérer que les plans climat territoriaux ont peu ou pas développé de prospective énergie climat. S'ils ne développent pas leur prospective énergétique, les PCET risquent de réduire l'objectif de facteur 4 à un simple slogan, sans que cet objectif soit assimilé par sa traduction en orientations ou actions concrètes. Le risque est alors de ne pas envisager une succession ou à une combinaison de crises économiques, sociales, environnementales, découlant de contraintes énergie-climat. Seuls quelques acteurs dont les mouvements écologistes et certains acteurs économiques dotés de fortes capacités de prospective comme la sidérurgie (Rynikiewicz, 2007) sont aujourd'hui convaincus qu'il faut élaborer différents scénarios permettant d'identifier et de préparer les mutations structurelles nécessaires. Il semble donc important que les élus investissent le domaine de la prospective énergétique et s'approprient les réflexions sur la résilience de leur territoire à ces nouveaux défis.

Quelques travaux amorcent des analyses de moyen terme sur une base territoriale. C'est ainsi le cas des agglomérations de Lyon (scénarii climat vers une agglomération sobre en carbone) et de Nantes Métropole (scénarii Explicit à 2025). Le scénario de Virage Energies Nord-Pas de Calais (Le Saux, 2008) est original en ce qu'il se caractérise par une construction collective et bénévole, qui suscite d'autres vocations d'associations à travers le territoire, notamment en Bretagne, et en Île de France. Il dénote une appropriation citoyenne émergente du facteur 4.

Quant au long terme, les collectivités se heurtent à la difficulté de penser ce changement de paradigme vers des villes post-carbone. La réflexion se limite encore au cercle des chercheurs et initiés. De plus, ces travaux de prospective climatique locale ne font que démarrer et produisent actuellement des catégories de scénarios possibles (Theys, 2009). Développer des «PCET de $2^{e}$ génération ", suppose de s'appuyer sur ces scénarios pour nourrir un plan d'actions court terme et justifier des orientations pour la planification des politiques publiques. Pour cela, les collectivités ont besoin que l'État accélère le 
travail encore récent mené au niveau national et le complète (scénarios officiels facteur 4 2050). Il est en effet nécessaire de raisonner au-delà de l'échelle locale pour intégrer les contraintes macroscopiques imaginables. De même que les modèles du GIEC servent de référence quant aux possibles évolutions du climat, l'État pourrait construire un éventail de scénarios nationaux balayant les possibles, allant de la prolongation de tendances actuelles à des scénarios de rupture claire. Certains des scénarios qui cherchent à anticiper les crises énergétiques et climatiques annoncées par les experts devraient notamment se fonder sur un prix du pétrole durablement supérieur à 100 dollars le baril. On peut également faire l'hypothèse d'un nouvel accord climatique international postKyoto, qui pousse l'Europe ou la France à imposer une contrainte carbone forte (taxe carbone, quotas de $\mathrm{CO}_{2}$, carte $\mathrm{CO}_{2}$ individuelle...), même en l'absence de crise énergétique. À ce cadre réglementaire et économique s'ajouteraient les possibles évolutions technologiques et sociologiques.

Cependant, alors même qu'une vision prospective est aujourd'hui indispensable, la prospective publique est en crise. L'enjeu pour l'État n'est plus de créer des scénarios, qui existent pour l'essentiel, mais de les rassembler dans un panel ayant valeur de référence pour les services déconcentrés de l'État qui auront à les promouvoir. Un débat national donnerait aux différentes écoles de pensée le lieu pour débattre des hypothèses sousjacentes. Ainsi, un grand nombre de scénarios sont fondés sur les projections de prix du pétrole établies par l'Agence Internationale de l'Energie (AIE), qui minore depuis longtemps la rapidité d'épuisement des réserves d'énergie fossiles et l'augmentation attendue des prix à moyen terme. Ce n'est ainsi qu'en 2008 que l'AIE a révisé de 30 à 70 dollars le prix tendanciel du baril en 2030, la même année où le prix réel était monté à plus de 150 dollars/Bl. Ce panel de cinq à dix scénarios nationaux inclurait une trajectoire « laisser-faire », fondée sur la prolongation des tendances actuelles, et plusieurs scénarios avec contraintes énergie climat plus élevées, compatibles ou non avec le facteur 4 , dans une logique de contraintes subies ou choisies. Afin de souligner les marges de choix dont disposent les citoyens et les élus, il semble préférable que l'approche prospective soit, dans un premier temps, exploratoire et non normative.

Les collectivités, avec l'éventuel appui de l'État, auront ensuite à mettre en débat auprès des partenaires de leur plan climat ces futurs possibles. «En contribuant à articuler les questions sur les avenirs possibles et celles sur les avenirs souhaitables pour un territoire, la prospective contraint les instances du pouvoir à se situer et jette les bases de nouvelles formes de dialogue social. La diffusion des travaux prospectifs joue un rôle décisif dans le changement des comportements et des mentalités.» (Durance, 2007 : 14). Les collectivités peuvent donc contribuer à l'acceptabilité sociale de tels scénarios en France. Elles auront à les vulgariser pour illustrer l'ampleur de la transition énergétique requise et à animer la construction collective d'imaginaires locaux (villes post-carbone ou territoire zéro fossiles, $100 \%$ énergies renouvelables, etc.). Ces débats ne seront pas aisés tant d'une part, la remise en cause est majeure est d'autre part, la société préfère globalement le statu quo. On peut prévoir une polarisation croissante des débats en France entre d'une part des critiques radicaux des sociétés de consommation, d'autre part des segments plus conservateurs de la société ou leurs représentants. Par contraste, ces débats sont mieux assumés dans des pays scandinaves ou anglo-saxons: Suède sans pétrole en 2020 (Commission on Oil Independence, 2006), villes en transition anglaises, ou même programme d'écologie radicale commandé... par le parti conservateur britannique (Goldsmith, Gummer, 2007). 


\section{Conclusion} qui permette de le populariser. Mais s'il est présent dans les objectifs de la première génération de PCET, il ne s'incarne pas encore dans leurs plans d'action. L'acceptabilité de tels plans d'action interroge leurs pilotes. Pour affronter cette difficulté, la seconde génération de plans climat aura besoin de confier aux élus une place plus importante. Ils devront convaincre leurs concitoyens que le défi est considérable mais nécessaire. Les techniciens auront à mener de front des actions sur les trois périmètres d'action de la collectivité : l'interne, les politiques publiques, l'externe. Ils auront à gérer les exercices simultanés de prospective, de planification et de mise en œuvre d'actions. Il reviendra aux politiques de faire adhérer leurs acteurs territoriaux à quelques futurs souhaitables, après avoir partagé des choix sur les contraintes externes potentielles.

point crucial des plans climat dans leur prochaine phase sera leur capacité à mettre en mouvement les acteurs du territoire. Il faudra placer ces partenaires en capacité d'agir (formations...) et de définir collectivement, au plus près de la population, un nouveau cadre de référence sur les droits de chacun à polluer l'air et à consommer de l'énergie. Enfin, il faudra tenir compte des limites qui découlent du partage de ces droits au niveau local, en accord avec le niveau mondial.

Il est en effet très souhaitable que les PCET de seconde génération mettent en place les mesures structurelles qui faciliteront l'intégration des acteurs locaux à une société sous contrainte carbone et énergétique bien plus forte que celle d'aujourd'hui.

\section{BIBLIOGRAPHIE}

ADEME, 2005, Guide : Un plan climat à l'échelle de mon territoire, http://www2.ademe.fr/servlet/ getDoc?sort=-1\&cid=96\&m=3\&id=39672\&ref=12441\&nocache=yes\&p1=111

ADEME, 2006, Expérimentation Bilan Carbone ${ }^{\oplus}$-Collectivités - Synthèse du retour d'expérience, http:// www2.ademe.fr/servlet/getBin?name=09761F4CE2918B91069913446E1F314B1165221520481.pdf

ADEME, 2009, Construire et mettre en œuvre un plan climat territorial : guide méthodologique,

http://www2.ademe.fr/servlet/getDoc?

sort $=1 \&$ cid $=96 \& m=3 \& i d=61443 \&$ ref $=\&$ nocache $=$ yes $\&$ p $1=111$

Association NégaWatt, 2005, Scénario négaWatt 2006 : pour un avenir énergétique sobre, efficace et renouvelable, Paris

Algan Y., Cahuc P., 2007, La société de défiance, comment le modèle social français s'autodétruit , Centre pour la recherche économique et ses applications (CEPREMAP), Paris, ENS Éditions

Baer P., Athanasiou T., Kharta S., 2008, The Greenhouse Development Rights Framework, Heinrich Boell Stiftung, Berlin

Développement durable et territoires, Vol. 2, n 1 | Mars 2011 
Berger G., 1967, Étapes de la prospective, PUF, Paris

Berthier J., Richard M., 2010, Comment réduire les émissions de gaz à effet de serre au niveau local, Outil pédagogique, Réseau Action Climat France, Montreuil

Boutaud, A., 2009, Les agendas 21 locaux, Bilan et perspectives en Europe et en France, commande pour le Grand Lyon, Centre de Ressources Prospectives du Grand Lyon, http://www.millenaire3.com/ uploads/tx_ressm3/Agenda21.pdf

Bozonnet, J.-P., 2009, « Attitudes face à l'environnement et cultures politiques en Europe », in Les Cahiers de l'IAU Île de France, ${ }^{\circ}$ 152, « Composer avec l'environnement », p. 10-11, http://www.iauidf.fr/fileadmin/Etudes/etude_630/

cahier_152_composer_avec_environnement.pdf\#pagemode=bookmarks\&page $=12$

Clerc D., Chalon C., Magnin G., Vouillot H., 2008, Pour un nouvel urbanisme, la ville au cœur du développement durable, éditions Yves Michel, collection société civile, Gap

Commissariat général du Plan, 1972, Plan et prospective, l'énergie, Armand Colin, Paris

Commissariat général du Plan, 1998, Énergie 2010 2020. Les défis du long terme, Lavoisier, Paris

Commission on Oil Independence, 2006, Making Sweden an OIL-FREE Society,

http://www.sweden.gov.se/sb/d/574/a/67096;jsessionid=acWDMAUu0up5

Cordeau E., Cassin P., 2009, «Air, énergie, climat : une planification évolutive », in Les Cahiers de l'IAU Île-de-France, n 152, « Composer avec l'environnement », p. 32-33, http://www.iau-idf.fr/ fileadmin/Etudes/etude_630/cahier_152_composer_avec_environnement.pdf

De Boissieu C., 2006, Rapport du groupe de travail « Division par quatre des émissions de gaz à effet de serre de la France à l'horizon 2050 ", Ministère de l'industrie et Ministère de l'écologie et du développement durable, La Documentation française, Paris

Durance P., 2007, « Planification, prospective et aménagement du territoire, Une genèse de la prospective territoriale en France », Lipsor, CNAM Paris, août

Énergie Cités, 2004, Historique énergie de la ville de Besançon, http://www.energie-cites.eu/db/ besancon_563_fr.pdf

ENERDATA-LEPII, 2005, Étude pour une prospective énergétique concernant la France, réalisée pour l'Observatoire de l'Énergie, Direction Générale de l'Énergie et des Matières Premières, http://upmfgrenoble.fr/iepe/textes/EPE_Enerdata-etude-facteur-quatre.pdf

ETD, 2009, La prise en compte de l'énergie et du climat dans les SCoT, http://

www.projetdeterritoire.com/index.php/Espaces-thematiques/Gestion-de-l-espace/Actualites/ Quelle-prise-en-compte-de-l-energie-et-du-climat-dans-les-SCoT

Gaudin T., 2005, La prospective, PUF, Collection Que sais-je, Paris

Global Chance, 2003, Petit mémento énergétique, les projections énergétiques françaises, http:// www.global-chance.org/IMG/pdf/GCnHS1p18-19.pdf

Goldsmith Z., Gummer J., 2007, Blueprint for a green economy, Conservative Party, Londres Grand Lyon, 1998, Charte de l'écologie urbaine, présentation et fiches action, 2 tomes.

IPCC, 2007, Summary for Policymakers, in Climate Change 2007 : Mitigation, contribution of Working Group III to the Fourth Assessment Report of the Intergovernmental Panel on Climate Change (B. Metz, O.R. Davidson, P.R. Bosch, R. Dave, L.A. Meyer eds), Cambridge University Press, Cambridge Lenoir D., 2007, Énergie Changeons de cap! Scénario pour une France durable, Terre Vivante, Mens 
Leray T, de La Roncière B., 2003, 30 ans de maîtrise de l'énergie, ATEE

Le Saux G., 2008, Énergies d'avenir en Nord-Pas de Calais, quelles solutions au dérèglement climatique, rapport complet version 1.0, Virage Énergie, Lille

Lorrain D., 2004, « Les pilotes invisibles de l'action publique, le désarroi du politique » in P. Lascoumes, P. Le Galès éd., Gouverner par les instruments, FNSP Les Presses de Science Po, Paris Meadows Do., Meadows De., Randers J., Behrens W., 1972, Halte à la croissance ? Rapport sur les limites de la croissance, traduction et édition française, 1973, édition Fayard, Paris

MEEDD, 2004, Plan climat 2004 : face au changement climatique, agissons ensemble.

MEEDD, 2006, Actualisation 2006 du Plan Climat 2004-2012, http://www.developpementdurable.gouv.fr/Les-plans-climat-2004-et-2006.html

MEEDDAT, 2009, Circulaire du 23 mars 2009 relative à la territorialisation de la mise en œuvre du Grenelle de l'environnement,

http://www2.equipement.gouv.fr/bulletinofficiel/fiches/Bo20098/met_20090008_0100_0022.pdf MEEDDM - Commissariat Général au Développement Durable, 2009, Vers une prospective territoriale post Grenelle de l'environnement : questions et modes d'emploi, Collection Études et documents, $\mathrm{n}^{\circ} 12$, http://www.developpement-durable.gouv.fr/IMG/pdf/E_D-12_cle1675c6_1_.pdf

MEEDDM, 2010, plan climat de la France, mise en œuvre du Grenelle Environnement, http:// www.developpement-durable.gouv.fr/Le-plan-climat-de-la-France.html

Meinshausen M., Meinshausen N., Hare W., Raper S. C. B., Frieler K., Knutti R., Frame

D. J., Allen M. R., 2009, “Greenhouse-gas emission targets for limiting global warming to $2^{\circ} \mathrm{C}$ ", Nature, Letters, 458, p. 1158-1162, http://www.nature.com/nature/journal/v458/n7242/abs/ nature08017.html

Métreau E, Tillerson K., 2007, "Local energy advice centres in France : quite active but how effective? An evaluation of local energy actions in France”, 2007 Summer Study - Saving Energy Just do it !, ECEEE, Stockholm

PUCA, 2009, L'habitat existant dans la lutte contre l'effet de serre : enquête qualitative sur les OPAH à volet énergie de nouvelle génération, http://rp.urbanisme.equipement.gouv.fr/puca/edito/ OPAH_energie.pdf

Radanne P., 2005, Énergies de ton siècle: des crises à la mutation, Lignes de repères, Gambais

RARE, 2004, Guide « Objectif développement durable : comprendre \& agir sur son territoire »- Retour d'expériences et recommandations pour l'agenda 21 local, http://www.rare.asso.fr/images/ guidedd_05.pdf

Rynikiewicz C., 2007, Contrainte d'environnement global et changement technique induit : vers des trajectoires d'innovations radicales dans la sidérurgie, thèse, Université Pierre-Mendès-France, Grenoble

Stern N., 2006, Rapport sur l'économie du changement climatique, rédigé pour le gouvernement du Royaume-Uni, résumé en français, http://www.hm-treasury.gov.uk/d/

stern_longsummary_french.pdf

Theys J., 2009, « Scénarios pour une ville post-carbone », Constructif, n² 23, http:// www.constructif.fr/Article_43_78_602/Scenarios_pour_une_ville_post_carbone.html Weizsäcker (von) E., Lovins A. B., Lovins L. H., 1997, Facteur 4 : deux fois plus de bien-être en consommant deux fois moins de ressources - Rapport au Club de Rome, Terre Vivante, Mens 


\section{RÉSUMÉS}

Si les chocs pétroliers initient les politiques d'économies d'énergie des collectivités françaises, ce n'est qu'un peu après le sommet de Kyoto, au tournant des années 2000, que naissent les plans climat, par lesquels elles s'engagent sur un objectif quantifié et daté de réduction des émissions. Ces plans climat énergie territoriaux de première génération intègrent souvent l'objectif facteur 4. En pratique, ils butent sur diverses difficultés de conception et de mise œuvre, reflétant un hiatus entre des plans d'actions de court terme, tendanciels et correctifs, et un objectif de long terme requérant de profondes mutations. L'articulation entre des plans d'action énergie-climat de court terme, la planification territoriale et le développement d'une prospective énergétique territoriale apparaît alors comme une condition d'appropriation et de succès essentiels du facteur 4 par les acteurs locaux.

While the first energy policies adopted by French local authorities resulted from the 1970s oil crises, the first local climate protection plans only started to emerge around 2000, in the wake of the Kyoto Earth Summit. These first generation plans are committed to a time-bound and measurable objective of emissions reduction and often refer to the factor 4 objective. They are faced with various conception and implementation challenges, which lead to a major discrepancy between short-term and corrective measures based on current trends, and a long-term objective requiring structural change. Combining short-term climate protection plan, land planning and the development of local energy futures studies thus appears as a key condition for local actors to truly work towards factor 4 .

\section{INDEX}

Keywords : climate change, climate protection plan, energy scenarios, factor 4, land planning, local authority, local agenda 21, sustainable development local process, local energy futures studies, transition cities

Mots-clés : changement climatique, démarche territoriale de développement durable, facteur 4, plan climat énergie territorial, planification territoriale, agenda 21 , prospective énergétique territoriale, prospective post Grenelle, scénarios climat énergie, villes post carbone

\section{AUTEUR}

\section{SYLVAIN GODINOT}

Sylvain Godinot, ingénieur en environnement, est responsable du pôle climat énergies à l'Agence Locale de l'Énergie de l'agglomération lyonnaise, d'où il accompagne le Grand Lyon et des communes dans leur plan climat. sylvaingodinot@altern.org 